\title{
CARACTERIZACIÓN FENO-GENOTÍPICA Y SIMBIÓTICA DE RIZOBIOS AISLADOS DE Desmanthus Virgatus (L.) WILLD. EN SUELOS DE LA PROVINCIA DE JUJUY (ARGENTINA)
}

\author{
ForNASERO, L.V. ${ }^{1} ;$ ZUBER, N. E. ${ }^{1,2} ;$ TONIUTTI, M. A. ${ }^{1}$ \\ Del Papa, M. F..$^{2}$ Pensiero, J. F. ${ }^{1}$ \& Lagares, A. ${ }^{2}$
}

\begin{abstract}
RESUMEN
Las leguminosas forrajeras nativas presentan un gran potencial productivo y un ejemplo de ellas es Desmanthus virgatus de amplia distribución en el centro norte de Argentina. El objetivo del trabajo fue realizar una caracterización feno-genotípica y simbiótica de rizobios que forman nódulos en $D$. virgatus con vista hacia el uso sustentable integral de dicha leguminosa. Para ello, se estableció una colección de 17 aislamientos recuperados de suelos de Jujuy. La caracterización fenotípica permitió reconocer rizobios de crecimiento rápido que mostraron un desarrollo óptimo entre pH 5 y 8, temperaturas de 28 a $35^{\circ} \mathrm{C}$, y en concentraciones de $1 \%(\mathrm{p} / \mathrm{v})$ de $\mathrm{NaCl}$. Se hallaron rizobios con capacidad de crecimiento en condiciones extremas de $\mathrm{pH} 9$ y a $40^{\circ} \mathrm{C}$. Los aislamientos se identificaron como pertenecientes a Ensifer spp. y los perfiles de amplificación de ADN genómico mostraron escasa diversidad genética. La inoculación de D. virgatus con la cepa 8L12.1 (Ensifer sp.) produjo incrementos de biomasa seca aérea de $73 \%$ en cámara de cultivo y $124 \%$ a campo respecto a las plantas sin inocular y sin fertilizar.
\end{abstract}

Palabras clave: microsimbiontes, leguminosas nativas, fijación biológica de nitrógeno.

1.- Facultad de Ciencias Agrarias, Universidad Nacional del Litoral. Kreder 2805. 3080HOF, Esperanza, Santa Fe, Argentina. Email: lfornase@fca.unl.edu.ar

2.- IBBM - Instituto de Biotecnología y Biología Molecular, CONICET - Departamento de Ciencias Biológicas. Facultad de Ciencias Exactas, Universidad Nacional de La Plata, Calles 47 y 115. (1900) La Plata, Argentina.

Manuscrito recibido el 22 de abril de 2020 y aceptado para su publicación el 20 de noviembre de 2020.

Fornasero LV, Zuber NE, Toniutti MA, Del Papa MF, Pensiero JF, Lagares A. Caracterización feno-genotípica y simbiótica de rizobios aislados de Desmanthus Virgatus (l.) Willd. en suelos de la provincia de Jujuy (Argentina). FAVE - Ciencias Agrarias 20 (1): 247-264. CC BY-NC-SA 4.0 


\begin{abstract}
Pheno-genotypic and symbiotic characterization of isolated rhizobia of Desmanthus Virgatus (I.) Willd. In soils of the province of Jujuy (Argentina)

Native forage legumes have great productive potential and an example of which is Desmanthus virgatus with a wide distribution in central and northern Argentina. The objective of the work was to carry out a pheno-genotypic and symbiotic characterization of rhizobia that form nodules in $D$. virgatus with a view to the integral sustainable use of said legume. For this, a collection of 17 isolates recovered from Jujuy soils was established. Phenotypic characterization allowed to recognize fast-growing rhizobia that showed an optimal development between $\mathrm{pH} 5$ y 8, temperatures to 28 a $35^{\circ} \mathrm{C}$, and in concentrations of $1 \%(\mathrm{w} / \mathrm{v})$ of $\mathrm{NaCl}$. Rhizobia with growth capacity were found under extreme conditions of $\mathrm{pH} 9$ and at $40^{\circ} \mathrm{C}$. The isolates were identified as belonging to Ensifer spp. and the genomic DNA amplification profiles showed little genetic diversity. The inoculation of $D$. virgatus with the strain 8L12.1 (Ensifer sp.) produced increases of 73\% aerial dry biomass in the cultivation chamber and $124 \%$ in the field with respect to the uninoculated and unfertilized plants.
\end{abstract}

Key words: microsymbionts, native legumes, biological nitrogen fixation.

\section{INTRODUCCIÓN}

Las leguminosas forrajeras nativas presentan un gran potencial productivo ya que constituyen excelentes fuentes de proteína $\mathrm{y}$ minerales para rumiantes en pastoreo, siendo un ejemplo de ellas las especies que pertenecen al género Desmanthus (Burt 1993). Desmanthus virgatus (lato sensu) es un complejo en el que actualmente se reconocen cuatro especies nativas del centro-norte argentino: $D$. acuminatus Benth., D. paspalaceus (Lindm.) Burkart, D. virgatus (L.) Willd. y D. tatuhyensis Hoehne (Luckow 1993, Zuloaga y Morrone 1999). Cabe destacar que, las tres primeras presentan mayor potencial forrajero debido a su foliosidad y capacidad de resiembra (Clem y Hall 1994, Jones y Brandon 1998, Zabala et al. 2008).
Desmanthus virgatus es una especie perenne, de ciclo primavero-estival, que se distingue por su alta palatabilidad y calidad del forraje (Jones y Clem 1997, Pengelly y Conway 2000). Si bien esta especie ha sido introducida con éxito al cultivo en sistemas pastoriles extensivos de Australia (Jones y Brandon, 1998), se reportaron síntomas de clorosis, poco vigor y escasa producción de plantas, problemas atribuidos a una inadecuada nodulación (Bahnisch et al. 1998). Como alternativa, se han empleado rizobios aislados de Leucaena leucocephala y Neptunia plena (Date 1991) como inoculantes de campo, con respuestas variables dependiendo del sitio de origen y de la presencia de rizobios en los suelos (Brandon et al. 1998). Existen experiencias en Australia que demuestran la necesidad de incluir la técnica de inoculación con cepas altamente efectivas en la rutina de siembra de $D$. virgatus (Bahnisch et al. 1998, Brandon et al. 1998, Gardiner 2016).

| Revista FAVE - Ciencias Agrarias 20 (1) 2021 
En las últimas décadas, diversos investigadores reportaron el gran potencial forrajero de Desmanthus spp. (Fischbach et al. 2005, Fontenele et al. 2009, Calado et al. 2016, Pensiero y Zabala 2017). Debido a ello, surgió la necesidad de investigar la naturaleza y características de los rizobios noduladores de estas leguminosas en el Continente Americano. En particular, Beyhaut et al. (2006a) caracterizaron la simbiosis y filogenia de los aislamientos de nódulos de D. illinoensis en Estados Unidos. En Argentina, Pedraza et al. (2001) describieron el fenotipo de rizobios nativos de D. virgatus, y posteriormente, Fornasero et al. (2014) aislaron y caracterizaron cepas simbiontes de $D$. paspalaceus recuperados de suelos de la provincia de Santa Fe.

El conocimiento y manejo agronómico de las interacciones simbióticas de poblaciones nativas y naturalizadas de rizobios es un factor clave en la dinámica de las leguminosas nativas en sistemas ganaderos, así como en el éxito de su introducción a cultivo y persistencia. En este contexto, la caracterización funcional, molecular y simbiótica de las poblaciones de rizobios nativos constituirá una valiosa herramienta hacia la selección de cepas con potencial para su utilización en inoculantes que permitan un mejor establecimiento de las leguminosas y con ello un incremento en la producción agropecuaria y una reducción del empleo de fertilizantes nitrogenados. Por todo lo expuesto, el objetivo de la presente investigación fue la caracterización fenogenotípica y la estimación de la fijación biológica de nitrógeno (FBN) en condiciones ambientales controladas y a campo de los rizobios simbiontes de Desmanthus virgatus.

\section{MATERIALES Y MÉTODOS}

\section{Sitio de muestreo y material colectado}

En base a la información suministrada por Zabala et al. (2008) se seleccionó y colectó una población de plantas de $D$. virgatus con suelo rizosférico en la localidad de Ledesma, provincia de Jujuy, Argentina (2345'40,1”S, 6443’21,8”O). Dicha población se documentó con un ejemplar que se herborizó y determinó taxonómicamente según Luchetti (2014) y se conservó bajo la colección de Pensiero 7059 (SF) en el Herbario "Arturo E. Ragonese" de la Facultad de Ciencias Agrarias (FCA), Universidad Nacional del Litoral (UNL). Asimismo, se colectaron los frutos maduros de 20-40 plantas de la población y se tomaron muestras de suelo.

El sitio de origen de la colecta corresponde, según Cabrera (1994), a la Provincia Fitogeográfica de las Yungas, Distrito de la Selva de Transición (Bosque Pedemontano), con suelos molisoles de textura franco-arcillosa (Visor GeoInta 2017) y clima cálido y húmedo, con precipitaciones estivales y heladas durante el invierno. Las características químicas del suelo fueron: 2,11\% de materia orgánica (Walkey y Black 1934), 0,10\% de nitrógeno orgánico total (Kjeldhal, Helrich 1990), 17 ppm de fósforo asimilable (Bray y Kurtz 1945), pH 6,9 (relación 2:1) (potenciometría Ministerio de Agricultura y Ganadería [MAG] 1982) y 0,105 dSm-1 de conductividad eléctrica (MAG 1982).

Las semillas recolectadas se acondicionaron y conservaron en el Banco de Germoplasma "Ing. Agr. José Mario Alonso" de la FCA- UNL y las plantas vivas se cultivaron en el Jardín de Introducción del Campo Experimental Juan Donnet de la FCA (312’'S, 6056’O). 


\section{V. Fornasero et al.}

\section{Evaluación de la nodulación rizobio - D. Características fenotípicas de los virgatus \\ aislamientos}

La evaluación de la nodulación se realizó a partir de plantas provenientes de campo y de aquellas que crecieron en cámaras de cultivo en condiciones ambientales controladas, con muestras de suelo y D. virgatus como plantas trampas. En los ensayos de laboratorio, las semillas que se colectaron en el campo se escarificaron según Hopkinson y English (2004). La desinfección se realizó por inmersión en etanol 96\% durante un minuto, 0,5\% (p/v) de hipoclorito de sodio cinco minutos y seis lavados con agua destilada estéril, y luego permanecieron una hora en el agua del último lavado. Las semillas se germinaron en condiciones asépticas sobre agar-agua 1\% $(\mathrm{p} / \mathrm{v})$ contenido en placas de Petri y luego se colocaron en macetas con vermiculita estéril y suelo en relación 5:1. Las plántulas permanecieron en cámaras de cultivo a $26^{\circ} \mathrm{C}$ de temperatura y fotoperiodo de $16 / 8$ horas (luz/oscuridad) durante 80 días.

Aislamientos de cepas bacterianas a partir de nódulos de plantas de $D$. virgatus

Los nódulos se desinfectaron superficialmente, se maceraron y los rizobios que contenían en su interior se aislaron en medio LMA (Extracto de Levadura-Manitol) con rojo Congo (Vincent 1970), hasta su purificación. Los aislamientos se confirmaron por su habilidad de formar nódulos en la planta huésped (Vincent 1970) y posteriormente se conservaron en caldo LMA con glicerol $50 \% \mathrm{v} / \mathrm{v}$ y se almacenaron a una temperatura de $-20^{\circ} \mathrm{C}$.

Las bacterias se cultivaron en LMA con rojo Congo a $28^{\circ} \mathrm{C}$ y se observó la morfología macroscópica de las colonias (Centro Internacional de Agricultura Tropical [CIAT] 1988). Las características morfológicas celulares y tinción diferencial de Gram se observaron a través de un microscopio óptico (Vincent 1970). La velocidad de crecimiento se evaluó según el tiempo de aparición de las colonias (Jordan 1984). Para analizar la respuesta al cambio de coloración del medio, cada aislamiento se cultivó en caldo LMA (pH 6,8) e indicador azul de bromotimol (0,5\% en $\mathrm{NaOH}$ $0,016 \mathrm{~N}$ ) a $28^{\circ} \mathrm{C}$ durante 5 días y se observó el cambio de coloración a azul (alcalinidad) o amarillo (acidez).

Capacidad de crecimiento in vitro de los aislamientos en diferentes condiciones de estrés abiótico

Los aislamientos se cultivaron en placas con LMA a $28^{\circ} \mathrm{C}, 35^{\circ} \mathrm{C}, 40^{\circ} \mathrm{C}$ y $45^{\circ} \mathrm{C}$. El crecimiento a diferentes niveles de $\mathrm{pH}$ y concentraciones de $\mathrm{NaCl}$, se determinó en LMA con el pH ajustado a 4, 5, 6, 7, 8 y 9; o con $0,01 \%$; 0,5\%; $1 \%$; $2 \%$ y $3 \%$ (p/v) de $\mathrm{NaCl}$. El crecimiento de las cepas en diferentes condiciones de temperatura, $\mathrm{pH}$ y concentraciones de $\mathrm{NaCl}$ se evaluó por duplicado a los 5 días y se calificó según una escala numérica gradual desde 0 (ausencia de crecimiento) hasta 5 (desarrollo normal). En todos los casos, la capacidad de crecimiento se comparó con el desarrollo bacteriano en medio LMA en condiciones óptimas ( $\mathrm{pH}$ $7 ; 28^{\circ} \mathrm{C}$ y $0,01 \%$ p/v $\mathrm{NaCl}$ ). Se utilizaron las cepas bacterianas de referencia: Ensifer meliloti 2011, Ensifer fredii USDA 205T, Rhizobium favelukesii LPU83, Rhizobium 
hainannense CCBAU 57015 (166), Rhizobium tropici IIBCIAT899 y Ensifer terangae CB3126.

\section{Características genotípicas de los rizobios}

Las preparaciones y manipulaciones de las muestras de ADN se realizaron según las técnicas descriptas en Sambrook et al. (1989).

Las huellas genéticas (fingerprints) de amplificación de ADN total se realizaron con los cebadores BOX A1R y MBOREP1 (Versalovic et al. 1994). Las condiciones de ciclado que se utilizaron fueron descriptas por Fornasero et al. (2014). Para el análisis de los productos de PCR se establecieron como presencia (1) o ausencia (0) de bandas para cada uno de los individuos analizados. Los datos se utilizaron para generar un dendograma UPGMA (coeficiente de similitud Dice). Se usaron los softwares Cross Checker, FAMD (Fingerprint Analysis with Missing Data) versión 1.23 beta (Schluter y Harris 2006) y MEGA 6 (Molecular Evolutionary Genetics Analysis) versión 6 (Tamura et al. 2013).

Aislamientos que se seleccionaron de la colección se analizaron por espectrometría de masa MALDI-TOF mediante el empleo del software MALDI Biotyper 3.1 (Bruker Daltonics, Bremen, Germany) (Maier et al. 2006). La preparación de las muestras se llevó a cabo de acuerdo con Toniutti et al. (2017). La identificación a nivel de género o especie de los espectros obtenidos se realizó según los valores de puntuación del software MALDI Biotyper: > 2 identificación a nivel de especie, entre 1,7 y 1,9 identificación a nivel de género y $<1,7$ identificación no confiable, según Ferreira et al. (2011).

\section{Ensayos de estimación de FBN en cámaras de crecimiento de cultivo en condiciones ambientales controladas}

Las semillas de $D$. virgatus cosechadas de las plantas cultivadas en el Jardín de Introducción de la FCA se escarificaron (Hopkinson y English, 2004) y se desinfectaron por inmersión en etanol 96\% durante un minuto, $0,5 \%(\mathrm{p} / \mathrm{v})$ de hipoclorito de sodio cinco minutos y seis lavados con agua destilada estéril. Luego, se germinaron en placas de Petri sobre agar-agua $1 \%(\mathrm{p} / \mathrm{v})$ y se colocaron individualmente en macetas de plástico que contenían vermiculita estéril y solución mineral de Jensen. Los tratamientos consistieron en testigo absoluto (plántulas sin inocular y sin fertilizar), testigo fertilizado (sin inoculación fertilizado con nitrógeno) e inoculado (inoculación con la cepa de rizobio a evaluar). La fertilización con nitrógeno $(\mathrm{N})$ se realizó con la adición de $70 \mathrm{ppm} \mathrm{N}\left(\mathrm{KNO}_{3}\right)$ y la inoculación se efectuó a los 15 días de la siembra mediante la aplicación de $10 \mathrm{~mL}$ de cultivo bacteriano ( $10^{7}$ ufc $\mathrm{mL}^{-1}$ solución Jensen). Las plantas permanecieron 80 días en condiciones de crecimiento controlado a $26^{\circ} \mathrm{C}$, fotoperiodo de $16 / 8$ horas (luz/oscuridad) y periódicamente se regaron con agua estéril y solución Jensen diluida 1:4. Se evaluó el número de nódulos y se determinó la materia seca (MS) de las plantas por secado en estufa a $65^{\circ} \mathrm{C}$ hasta peso constante. Los datos que se obtuvieron se analizaron según análisis de la varianza y test de Tuckey $(\mathrm{p}<0,01)$ mediante la utilización del software InfoStat (Di Rienzo et al. 2018). 


\section{Evaluación de los rizobios seleccionados simbiontes de D. virgatus en condiciones de campo}

Las cepas de rizobios que se seleccionaron por su mayor capacidad potencial de FBN se evaluaron en ensayos de parcelas a campo. La experiencia se llevó a cabo en el Campo Experimental Juan Donnet de la FCA -UNL, localidad de Esperanza

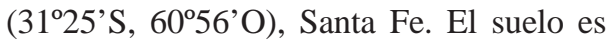
un Argiudol típico con textura franco limosa (Instituto Nacional Tecnología Agropecuaria [INTA], 1991) y las características químicas fueron: 2,4\% de materia orgánica (Walkley y Black 1934), 0,145\% de $\mathrm{N}$ total (Helrich 1990), 25 ppm de fósforo extractable (Bray y Kurtz 1945), pH 6,3 (relación 2:1) (MAG 1982) y 0,7 dSm-1 de conductividad eléctrica (MAG 1982). Se tomaron muestras de suelo y se realizó un recuento de rizobios por el método del Número más Probable (NMP) (Vincent 1970).

El ensayo se implantó en el mes de enero sembrando 100 semillas metro ${ }^{-1}$ lineal de D. virgatus cv Marc. Previamente, las semillas fueron escarificadas (Hopkinson y English, 2004). Los tratamientos fueron:

1.- control absoluto (sin inocular y sin fertilizar)

2.- inoculado con una cepa seleccionada de la colección

3.- inoculado con la cepa de referencia E. terangae CB3126

\section{4.- fertilizado con $\mathrm{N}$}

El diseño experimental fue en bloques completos al azar con tres repeticiones. Las parcelas consistieron en 4 surcos separados 0,15 m entre ellos y un metro de largo, y se separaron entre sí mediante la siembra de Setaria itálica con una densidad de 20 kg ha-1.
Los inoculantes se prepararon en turba estéril a partir de cultivos puros de rizobios y se efectuaron recuentos por el método de Recuento en Placa (Vincent 1970) para los inoculantes y semillas de $D$. virgatus inoculadas. En los tratamientos inoculados, las semillas se humectaron con una solución de sacarosa estéril 20\% (p/v) como adherente e inocularon con $8 \mathrm{~g}$ de inoculante $\mathrm{kg}^{-1}$ de semilla. En el tratamiento con $\mathrm{N}$, se iniciaron las fertilizaciones a los 15 días posteriores a la fecha de siembra y se aplicaron $20 \mathrm{~kg}$ de $\mathrm{N} \mathrm{ha}^{-1}$ cada dos semanas.

A las 15 semanas posteriores a la siembra se tomaron muestras de plantas de las líneas centrales de las parcelas y se determinaron MS vegetal por secado en estufa a $65^{\circ} \mathrm{C}$ hasta peso constante y contenido de $\mathrm{N}$ total en parte aérea de las plantas, según el método Kjeldahl (Helrich 1990). Se determinaron el Índice de efectividad de la inoculación (IEI) e Índice de respuesta al nitrógeno (IRN), según (CIAT 1988). Los datos de MS y contenido de $\mathrm{N}$ de las plantas se analizaron según análisis de la varianza y test de Tuckey $(p<0,01)$ mediante la utilización del software InfoStat (Di Rienzo et al. 2018).

\section{RESULTADOS Y DISCUSIÓN}

\section{Evaluación de la nodulación rizobio - D.} virgatus

Las plantas de $D$. virgatus procedentes de su hábitat natural presentaron un gran desarrollo del xilopodio con un estado avanzado de lignificación y escasas raíces secundarias. El número de nódulos fue entre 1 y 5 por ejemplar, bajo, en comparación con los estándares de nodulación que se observaron en leguminosas forrajeras (CIAT 1988). 
Además, los nódulos mostraron un tamaño pequeño, forma cilíndrica aplanada y color marrón oscuro, características que no coinciden con el aspecto típico para nódulos en activa fijación de N (Perticari 2006).

En cámara de crecimiento de cultivo, las plantas presentaron nódulos de tamaños diferentes, con una abundancia de categoría escasa a mediana, según los códigos de nodulación (CIAT 1988). Las estructuras nodulares de $D$. virgatus presentaron una forma alargada y a veces ramificada, que mostraron características típicas de crecimiento indeterminado y su coloración presentó variaciones desde rosado a pardo claro u oscuro.

\section{Colección de rizobios simbiontes de D. virgatus. Características fenotípicas de los aislamientos}

A partir de los nódulos se realizaron aislamientos y se obtuvieron 17 rizobios simbiontes de $D$. virgatus. Los aislamientos bacterianos presentaron colonias circulares de colores rosados o blanquecinos, translúcidas u opacas y la consistencia predominante fue mucilaginosa con producción de exopolisacáridos (EPS). La forma, transparencia y diámetro de las colonias de los rizobios depende en gran parte de la producción de EPS (Martins et al. 1997). Los rizobios mostraron colonias circulares de consistencia acuosa al inicio del crecimiento, que se tornaron en formas irregulares a medida que se producía mayor gomosidad durante el período de incubación. Martins et al. (1997), sugirieron que las colonias de rizobios presentan forma variable dependiendo de la consistencia de los EPS.

La morfología celular de los aislamientos se observó al microscopio óptico y los simbiontes presentaron forma de bacilos pequeños, Gram negativos y no esporula- dos, que corresponden a la descripción de bacterias que forman nódulos con leguminosas en el Phylum B12 Proteobacteria (Garrity et al. 2005).

Los rizobios desarrollaron colonias visibles con un diámetro igual o superior a 2 mm en medio LMA entre los 2 a 4 días posteriores al aislamiento. Además, los simbiontes acidificaron el medio de cultivo, ya que ocasionaron el cambio de coloración de verde a amarillo. Consistente con estas evidencias, López et al. (2000) y Teamtisong (2000) aislaron rizobios de crecimiento rápido de nódulos de $D$. virgatus. Asimismo, en la provincia de Tucumán, Pedraza et al. (2001) obtuvieron rizobios nativos de $D$. virgatus cv. Jaribu que crecieron en forma rápida, acidificaron el medio de cultivo y produjeron colonias muy gomosas.

Capacidad de crecimiento in vitro de los aislamientos en diferentes condiciones de estrés abiótico

La capacidad de los microorganismos para crecer en diferentes condiciones de cultivo puede variar ampliamente, incluso entre cepas de la misma especie. En el caso de los rizobios, se observaron diferencias entre cepas de un mismo género en relación a la tolerancia a temperaturas extremas, salinidad, acidez y alcalinidad (Amarger 2001).

El crecimiento de los rizobios simbiontes de $D$. virgatus que se evaluaron bajo diferentes condiciones abióticas en medio LMA se presenta en la Figura 1. En relación con la temperatura, los aislamientos mostraron crecimiento normal a $35^{\circ} \mathrm{C}$ y los simbiontes nativos 8L12.1, 8L12.3 y 8L14.1 fueron capaces de crecer a $40^{\circ} \mathrm{C}$, una temperatura registrada con frecuencia en las zonas de adaptación de $D$. virgatus. La mayoría de los rizobios son incapaces de crecer a $37^{\circ} \mathrm{C}$ (Graham 1992), temperatura 
que permite establecer diferencias entre algunas especies del género Rhizobium que forman nódulos en Phaseolus vulgaris (Ramírez-Bahena et al. 2008).

El crecimiento bacteriano para los géneros Mesorhizobium y Rhizobium se informó en un rango de pH 4 a 10 (Jarvis et al. 1997, Kuykendall et al. 2005) y para Sinorhizobium entre pH 5 y 10,5 (de Lajudie et al. 1994). Los rizobios simbiontes de $D$. virgatus presentaron un desarrollo óptimo en un intervalo de pH 5 a 8, y el 70\% de los

\begin{tabular}{|c|c|c|c|c|c|c|c|c|c|c|c|c|c|c|c|}
\hline \multirow{2}{*}{ Cepas } & \multicolumn{4}{|c|}{$\begin{array}{l}\text { Crecimiento a la } \\
\text { temperatura indicada }\left({ }^{\circ} \mathrm{C}\right)\end{array}$} & \multicolumn{6}{|c|}{ Crecimiento al nivel de pH indicado } & \multicolumn{5}{|c|}{$\begin{array}{l}\text { Crecimiento a la concentración } \\
\text { de } \mathrm{NaCl}\left(\% \mathrm{p}^{\prime} / v\right) \text { indicada }\end{array}$} \\
\hline & $28^{\circ}$ & $35^{\circ}$ & $40^{\circ}$ & $45^{\circ}$ & 4 & 5 & 6 & 7 & 8 & 9 & $0.01 \%$ & $0.5 \%$ & $1 \%$ & $2 \%$ & $3 \%$ \\
\hline $8\llcorner 2.2$ & 5 & 5 & 1 & 0 & 1 & 4 & 5 & 5 & 4 & 3 & 5 & 5 & 4 & 2 & 0 \\
\hline $8 \mathrm{~L} 4.2$ & 5 & 5 & 1 & 0 & 1 & 4 & 5 & 5 & 4 & 3 & 5 & 5 & 4 & 3 & 0 \\
\hline $8 \mathrm{~L} 4.5$ & 5 & 5 & 1 & 0 & 1 & 4 & 5 & 5 & 4 & 3 & 5 & 5 & 4 & 3 & 0 \\
\hline $8 \mathrm{~L} 4.9$ & 5 & 5 & 1 & 0 & 1 & 4 & 5 & 5 & 4 & 3 & 5 & 5 & 4 & 1 & 0 \\
\hline $8 \mathrm{~L} 6.3$ & 5 & 5 & 1 & 0 & 1 & 4 & 5 & 5 & 4 & 3 & 5 & 5 & 4 & 1 & 0 \\
\hline $8 \mathrm{~L} 10.1$ & 5 & 5 & 2 & 0 & 1 & 4 & 5 & 5 & 5 & 4 & 5 & 5 & 4 & 1 & 0 \\
\hline 8L11.1 & 5 & 5 & 2 & 0 & 1 & 4 & 5 & 5 & 5 & 4 & 5 & 5 & 4 & 1 & 0 \\
\hline $8 \mathrm{~L} 12.1$ & 5 & 5 & 5 & 0 & 1 & 4 & 5 & 5 & 5 & 4 & 5 & 5 & 4 & 1 & 0 \\
\hline $8 \mathrm{~L} 12.2$ & 5 & 5 & 2 & 0 & 1 & 4 & 5 & 5 & 5 & 4 & 5 & 5 & 4 & 1 & 0 \\
\hline $8 \mathrm{~L} 12.3$ & 5 & 5 & 5 & 0 & 1 & 4 & 5 & 5 & 5 & 4 & 5 & 5 & 4 & 1 & 0 \\
\hline $8 \mathrm{~L} 12.4$ & 5 & 4 & 3 & 0 & 1 & 4 & 5 & 5 & 5 & 4 & 5 & 5 & 3 & 1 & 0 \\
\hline $8 \mathrm{~L} 13.1$ & 5 & 5 & 3 & 0 & 1 & 4 & 5 & 5 & 5 & 4 & 5 & 5 & 4 & 1 & 0 \\
\hline $8 \mathrm{~L} 13.3$ & 5 & 5 & 2 & 0 & 1 & 4 & 5 & 5 & 5 & 4 & 5 & 5 & 4 & 1 & 0 \\
\hline $8 \mathrm{~L} 14.1$ & 5 & 5 & 4 & 0 & 1 & 4 & 5 & 5 & 5 & 4 & 5 & 5 & 4 & 1 & 0 \\
\hline 8L14.2 & 5 & 4 & 2 & 0 & 1 & 4 & 5 & 5 & 5 & 4 & 5 & 5 & 3 & 2 & 0 \\
\hline 8L15.1 & 5 & 4 & 2 & 0 & 1 & 4 & 5 & 5 & 5 & 4 & 5 & 5 & 4 & 1 & 0 \\
\hline 8L15.2 & 5 & 5 & 1 & 0 & 1 & 4 & 5 & 5 & 5 & 4 & 5 & 5 & 4 & 1 & 0 \\
\hline $\begin{array}{c}\text { E. melibtizo11 } \\
\text { E. fredï }\end{array}$ & 5 & 5 & 3 & 0 & 0 & 2 & 5 & 5 & 5 & 4 & 5 & 5 & 4 & 3 & 2 \\
\hline USDA205T & 5 & 5 & 0 & 0 & 1 & 3 & 5 & 5 & 5 & 4 & 5 & 5 & 4 & 1 & 0 \\
\hline $\begin{array}{l}\text { R. favelukess } i \\
\text { LPU83 } \\
R \text { hananense }\end{array}$ & 5 & 2 & 0 & 0 & 0 & 4 & 5 & 5 & 3 & 2 & 5 & 1 & 1 & 1 & 0 \\
\hline $\begin{array}{l}\text { CCBAU } 57015 \\
\text { R. tropici }\end{array}$ & 5 & 5 & 0 & 0 & 4 & 5 & 5 & 5 & 5 & 4 & 5 & 5 & 5 & 4 & 0 \\
\hline IBBCIAT899 & 5 & 5 & 1 & 0 & 4 & 5 & 5 & 5 & 5 & 4 & 5 & 5 & 5 & 4 & 3 \\
\hline $\begin{array}{l}\text { S. terangae } \\
\text { CB3126 }\end{array}$ & 5 & 5 & 4 & 0 & 0 & 4 & 5 & 5 & 5 & 4 & 5 & 4 & 2 & 1 & 0 \\
\hline
\end{tabular}

Figura 1: Crecimiento de rizobios aislados de D. virgatus en suelos de la provincia de Jujuy evaluados bajo diferentes condiciones de estreses abióticos en medio LMA. Escala de graduación: desde 5 hasta 0 indican la capacidad de los rizobios para crecer bajo la condición investigada (5: desarrollo óptimo, 0: ausencia de crecimiento)

Figure 1: Growth of rhizobia isolated from D. virgatus in soils of the provinces of Jujuy evaluated under different abiotic stressing conditions in agarized LMA medium. Scores from 5 to 0 indicate the ability of rhizobia to grow under the investigated condition (5:full development, 0:absence of growth). 
aislamientos fue capaz de crecer hasta $\mathrm{pH}$ 9. En este sentido, la tolerancia a diferentes niveles de $\mathrm{pH}$ es una característica deseable para las cepas inoculantes potencialmente comerciales (Soares et al. 2014, Hernández-Forte et al. 2017).

Los rizobios pueden tolerar concentraciones de sal más elevadas que sus plantas huéspedes. El aumento de la concentración de sal tiene un efecto perjudicial en la población microbiana del suelo como resultado de la toxicidad directa y el estrés osmótico (Carranca 2013). En este trabajo, la mayoría de los rizobios presentó un crecimiento óptimo en presencia de $1 \%$ de $\mathrm{NaCl}(\mathrm{p} / \mathrm{v})$, mientras que ningún aislamiento fue capaz de crecer en concentraciones superiores de sal.

\section{Características genotípicas de los rizobios}

El análisis de la diversidad genotípica del germoplasma asociado a $D$. virgatus, que se realizó a través de la generación de huellas digitales de ADN, reveló tres tipos principales de perfiles BOX A1R diferentes (similitud de 75\% para el valor de corte), según se muestra en la Figura 2. El perfil tipo "B" incluye el 77\% de los aislamientos, mientras que los perfiles “ $\mathrm{A}$ ” $\mathrm{y}$ " $\mathrm{C}$ ” se representan por una y dos cepas, respectivamente.

Los perfiles de amplificación de $\mathrm{ADN}$ genómico de las cepas simbiontes que se realizaron con el cebador MBOREP-1 presentaron resultados análogos a los obtenidos con BOX A1R, los cuales evidenciaron una escasa diversidad genética. Estos resul-

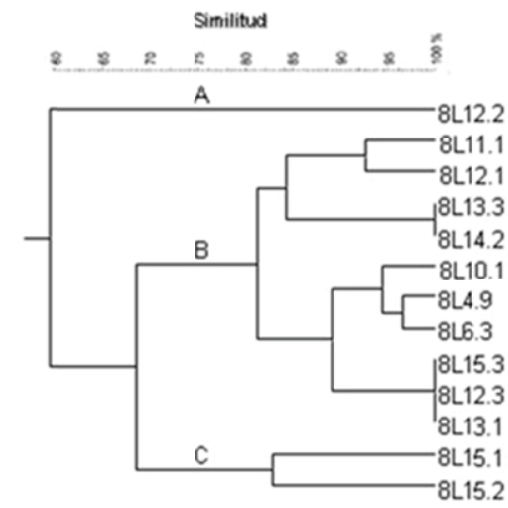

Figura 2: Análisis UPGMA obtenido en base a la presencia-ausencia de bandas en los productos de amplificación BOXA1R de diferentes aislamientos noduladores de D. virgatus recuperados de suelos de la provincia de Jujuy. La figura muestra el análisis UPGMA (coeficiente de similitud de Dice) de los productos BOXA1R-PCR obtenidos con el ADN total de los aislamientos indicados. Figure 2: UPGMA analysis obtained from the presence-absence of bands in the BOXA1R amplification products of different isolates nodulators of D.virgatus retrieved from soils in the provinces of Jujuy. The figure shows the UPGMA analysis (Dice similarity coefficient) derived from BOXA1RPCR products obtained with total DNA from the indicated isolates. 
tados difieren con investigaciones que demostraron una marcada diversidad genética en el germoplasma asociado a las leguminosas nativas $D$. paspalaceus (Fornasero et al. 2014), Desmodium incanun (Toniutti et al. 2017) y Macroptilium erythroloma (Fornasero et al. 2018) procedentes del centro y norte de nuestro país.

El análisis que se realizó por espectrometría de masas MALDI-TOF permitió genotipificar a los aislamientos 8L6.3, 8L12.1 y 8L14.2 simbiontes de $D$. virgatus como Ensifer spp. Anteriormente, Sinsuwongwat et al. (2002) reportaron el aislamiento de cepas de Rhizobium leguminosarum de nódulos de las raíces de $D$. virgatus en Tailandia. Beyhaut et al. (2006a) informaron que Rhizobium giardinii y $R$. leguminosarum produjeron nódulos en Desmanthus illinoensis. Posteriormente, Fornasero et al. (2014) mostraron que simbiontes del género Rhizobium relacionados con $R$. alamii y $R$. mesosinicum y aislamientos de cepas de Mesorhizobium (cercano a M. plurifarium) formaron nódulos en plantas de $D$. paspalaceus en la provincia de Santa Fe, Argentina.

\section{Estimación de FBN en cámaras de crecimiento de cultivo en condiciones ambientales controladas}

Los aislamientos de la colección que se utilizaron en los ensayos de FBN se seleccionaron en función de las características fenotípicas, tolerancia a estreses abióticos (con prioridad en el factor alta temperatura) y perfiles de amplificación de ADN genómico.

Las plantas de $D$. virgatus que se inocularon con los rizobios 8L12.3, 8L10.1 y 8L12.1 formaron 6, 13 y 14 nódulos por planta respectivamente, con diferencias significativas entre el tratamiento inoculado con la cepa 8L12.3 y los restantes tratamientos. Las plantas que se fertilizaron con
$\mathrm{N}$ y las que se inocularon con los rizobios 8L10.1 y 8L12.1 presentaron una biomasa aérea que representó entre 57 y 62\% de la MS total, mientras que las plantas del tratamiento testigo absoluto y las inoculadas con 8L12.3 mostraron una tendencia al equilibrio entre el peso de raíces y el peso de tallos y hojas. Los resultados demostraron que la inoculación de $D$. virgatus con las cepas 8L12.1 y 8L10.1 produjo incrementos de MS aérea de 73\% y 40\% respectivamente, en comparación con las plantas sin inocular y sin fertilizar (Figura 3).

Diversas investigaciones mostraron que los patrones de respuesta a la inoculación con rizobios en cámara de crecimiento de cultivos fueron variables entre especies e incluso accesiones del género Desmanthus. Así, Date (1991) demostró que el efecto de la inoculación con rizobios simbiontes de Leucaena spp., Gliricidia sp., Neptunia spp., Desmanthus spp. y Prosopis chilensis fue diferente entre accesiones distintas del género Desmanthus. Posteriormente, Illarze Dive (2011) informó que rizobios inoculados en Desmanthus spp. también mostraron una respuesta variable entre las especies con aumentos significativos en la MS aérea de las plantas. En particular, Beyhaut et al. (2006b) obtuvieron incrementos de $100 \%$ de biomasa vegetal de D. illinoensis con respecto a plantas sin inocular.

\section{Evaluación de los rizobios seleccionados (de élite) simbiontes de D. virgatus en condiciones de campo}

En el presente trabajo, la población de rizobios del suelo compatibles con $D$. virgatus fue inferior a $10^{2} \mathrm{NMP} \mathrm{g}^{-1}$ de suelo seco. Deaker et al. (2004) informaron que la nodulación en suelos con bajas poblaciones de rizobios dependerá de una correcta inoculación con cepas efectivas. En este 


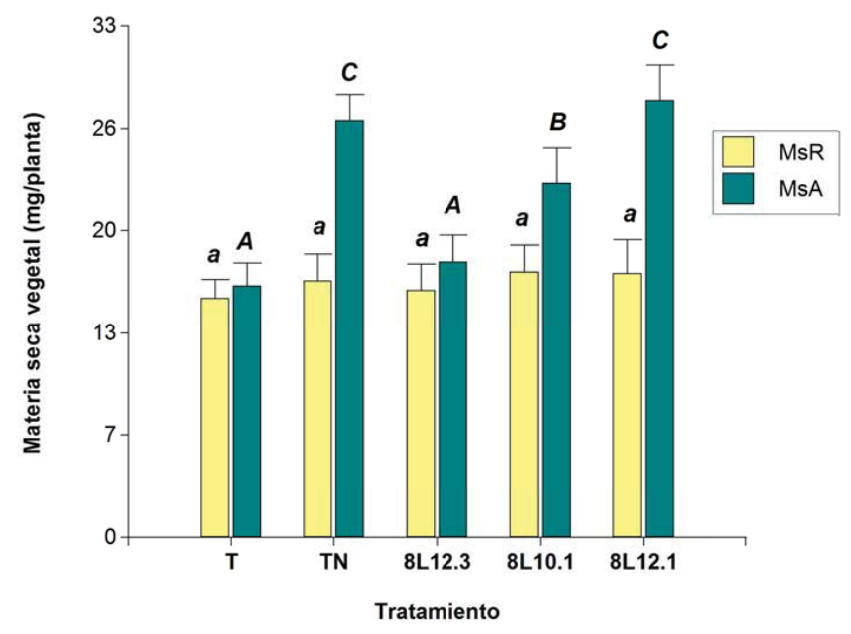

Figura 3: Efecto de la inoculación con rizobios presentes en suelos de Jujuy sobre la materia seca vegetal de D. virgatus en cámaras de crecimiento de cultivo. Las plantas crecieron en macetas con vermiculita y fueron inoculadas con los aislamientos indicados en la figura. Se incluyeron T: plantas sin inoculación y TN: plantas sin inoculación con nitrógeno. Tratamientos (n:10 a 12). Las barras corresponden a la desviación estándar de las medias. Letras diferentes indican diferencias significativas entre tratamientos según Test de Tukey $(p<0,01)$.

Figure 3: Effect of inoculation with rhizobia present in Jujuy soils on the plant dry matter of $D$. virgatus in culture growth chambers. The plants were grown in pots with vermiculite and were inoculated with the isolates indicated in the figure. T: plants without inoculation and TN: plants without inoculation with nitrogen were included. Treatments ( $n: 10$ to 12). The bars correspond to the standard deviation of the means. Different letters indicate significant differences between treatments according to Tukey's Test $(p<0.01)$.

sentido, la inoculación resultaría beneficiosa en sitios con una población de rizobios

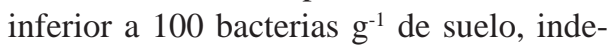
pendientemente de la eficiencia para fijar $\mathrm{N}$ de los rizobios nativos (Catroux et al. 2001, Howieson y Ballard 2004). Investigaciones realizadas por Bahnisch et al. (1998) demostraron que los suelos de Queensland podían contener pocos o ningún rizobio nativo capaz de formar nódulos en $D$. virgatus, sugiriendo la necesidad de disponer de cepas efectivas para su utilización como inoculantes biológicos.

La evaluación cuantitativa de los inocu- lantes mostró una carga bacteriana igual o superior a $1.10^{9}$ ufc g-1 $^{-1}$ de inoculante y $1.10^{4}$ ufc semilla ${ }^{-1}$. Los resultados mostraron que las concentraciones finales de los inoculantes cumplieron con las exigencias especificadas en las normativas de control de calidad de nuestro país (Puente et al. 2011).

En condiciones de campo, el efecto de la inoculación con las cepas de rizobios y la fertilización nitrogenada sobre la altura de las plantas de $D$. virgatus presentó variaciones desde 43,7 $\pm 10,3 \mathrm{~cm}$ para el control absoluto hasta 51,2 \pm 8,8 cm para las plantas fertilizadas, con diferencias significati- 


\section{V. Fornasero et al.}

vas entre ambos tratamientos. A su vez, la altura media de los ejemplares inoculados con el aislamiento 8L12.1 y con E. terangae CB3126 fueron similares, 49,5 \pm 9,0 cm y $47,7 \pm 8,6 \mathrm{~cm}$ respectivamente, sin mostrar diferencias estadísticas entre ellos $(p<0,01)$.

La respuesta a la inoculación con las cepas nativa 8L12.1 e introducida E. terangae CB3126 en la MS aérea y contenido de N de $D$. virgatus cv Marc, en condiciones de campo, se muestra en la Figura 4. La MS aérea de las plantas inoculadas con la cepa CB3126 fue superior a la MS del control absoluto, sin embargo, el valor fue significativamente menor que el de las plantas inoculadas con el aislamiento termotolerante 8L12.1 y de aquellas que se fertilizaron con N. Según datos de la literatura, la cepa comercial E. terangae CB3126 fue efectiva en la formación de nódulos y fijación de $\mathrm{N}$ en accesiones de $D$. virgatus cultivadas en macetas (Date 1991), y en D. virgatus cv Marc, D. leptophyllus cv Bayamo y D. pubescens cv Uman evaluados en ensayos a campo en Queensland (Brandon et al. 1998). Sin embargo, E. terangae CB3126

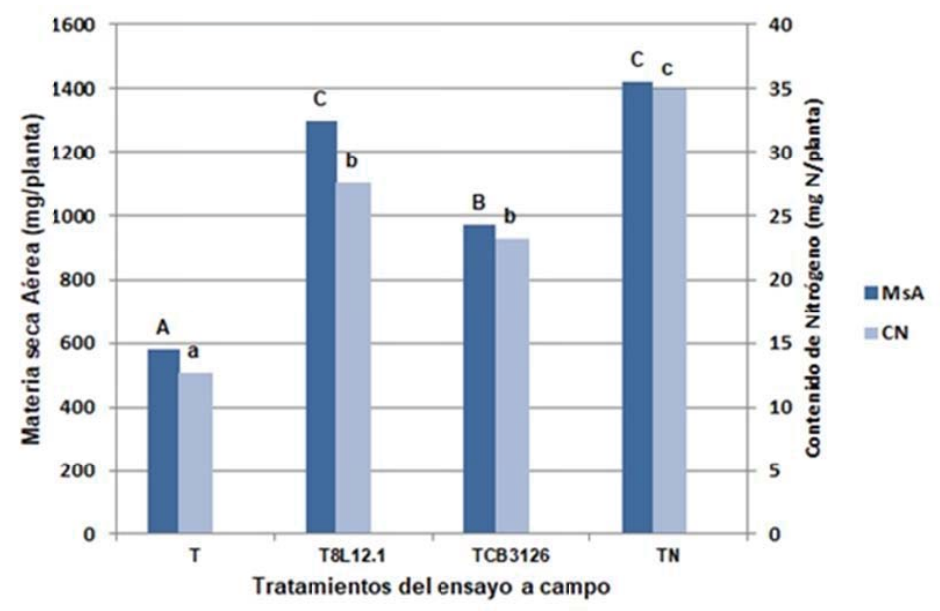

Figura 4: Efecto de la inoculación con cepas de rizobios sobre la materia seca aérea y contenido de nitrógeno de D. virgatus cv. Marc en condiciones de campo en la localidad de Esperanza, Santa Fe. T: plantas sin inoculación; T8L12.1: inoculadas con la cepa nativa 8L12.1; TCB3216: inoculadas con la cepa recomendada E. terangae CB3216; TN: sin inoculación con nitrógeno. Tratamientos (n:10 a 12). MsA: materia seca aérea por planta y CN: contenido de nitrógeno por planta. Letras diferentes indican diferencias significativas entre tratamientos según Test de Tukey $(p<0,01)$.

Figure 4: Effect of inoculation with rhizobia strains on the aerial dry matter and nitrogen content of D. virgatus cv. Marc in field conditions in the town of Esperanza, Santa Fe. T: plants without inoculation; T8L12.1: inoculated with the native strain 8L12.1; TCB3216: inoculated with the recommended strain E. terangae CB3216; TN: without inoculation with nitrogen. Treatments (n: 10 to 12). MsA: aerial dry matter per plant and CN: nitrogen content per plant. Different letters indicate significant differences between treatments according to Tukey's Test $(p<0.01)$. 
presentó una sobrevivencia muy baja en las semillas de $D$. virgatus almacenadas bajo condiciones cálidas y secas (Becerra Stiefel et al. 1998).

En las regiones del centro y norte de Argentina, las temperaturas elevadas características de la estación estival que se registran durante la siembra o en estadios iniciales del crecimiento vegetativo de las especies de Desmanthus, afectarían el contenido hídrico del suelo y provocarían condiciones estresantes para la sobrevivencia de las cepas simbiontes. En este contexto, el proceso de nodulación y FBN dependerá de la tolerancia a condiciones ambientales estresantes y de la efectividad de los rizobios nativos o introducidos con el inoculante biológico.

En la presente experiencia, la respuesta a la inoculación mostró incrementos de MS aérea de las plantas de $D$. virgatus de 67 y $124 \%$ en relación con las plantas sin inocular y sin fertilizar, para la cepa $E$. terangae CB3126 y el aislamiento 8L12.1 respectivamente. Evaluaciones de inoculación en cultivares comerciales de Desmanthus indicaron que aislamientos que se obtuvieron de L. leucocephala, E. terangae CB3126, mostraron incrementos de peso seco de las plantas de 34 a 313\%, dependiendo de la presencia de rizobios nativos y del contenido de $\mathrm{N}$ del suelo (Brandon et al. 1998).

Los contenidos de $\mathrm{N}$ en la biomasa aérea de las plantas inoculadas fueron significativamente superiores a los que se obtuvieron en las plantas testigo, lo que demostró la efectividad de las cepas utilizadas como inoculantes. El contenido promedio de proteína cruda (PC) en la MS de D. virgatus mostró valores de 14,5 hasta $18,0 \%$, para las plantas testigo, inoculadas y fertilizadas con $\mathrm{N}$. Investigaciones que se realizaron sobre el valor nutritivo de los cultivares de Desmanthus (Marc, Bayamo y Uman) y de la variedad ProgardesTM (D. bicornutus, D. leptophyllus y D. virgatus) mostraron contenidos de PC para la planta entera de 10,5 a 15,5\%, mientras que el mismo parámetro en hojas presentó un promedio de 20,2 a 22,4\% y en tallos de 7,1 a 11,9\% (Jones et al. 2000, Pengelly et al. 2006, Gardiner et al. 2013).

El IRN de las plantas de $D$. virgatus presentó un valor de 63, lo que demostró que los rizobios del suelo no mostraron una acción efectiva en el proceso simbiótico leguminosa-rizobio. Los valores de IEI de las plantas inoculadas con las cepas Ensifer sp. 8L12.1 y E. terangae CB3126 fueron 53 y 45 respectivamente, lo cual demuestra que las inoculaciones con rizobios presentaron un efecto positivo en la FBN con respecto a las plantas sin inocular. La respuesta al $\mathrm{N}$ sugiere la necesidad de efectuar la inoculación de las plantas de $D$. virgatus con rizobios fijadores de $\mathrm{N}$ bajo las condiciones de realización del ensayo. Asimismo, los resultados que se obtuvieron permitieron distinguir cepas de rizobios nativas con alta efectividad simbiótica para las plantas de D. virgatus.

\section{CONCLUSIONES}

Las plantas de $D$. virgatus procedentes de la localidad de Ledesma, provincia de Jujuy, presentaron estructuras nodulares de tamaño pequeño y color marrón oscuro, mientras que aquellas cultivadas con el suelo de origen en cámaras de crecimiento mostraron nódulos de tamaños variados y de crecimiento indeterminado, lo cual indica la presencia en el suelo de rizobios con 
capacidad de nodular Desmanthus. A partir de los nódulos obtenidos, se conformó una colección de rizobios simbiontes recuperados del suelo de origen en condiciones ambientales controladas.

La caracterización fenotípica de los simbiontes permitió reconocer rizobios de crecimiento rápido que mostraron un desarrollo óptimo en un rango de $\mathrm{pH}$ de 5 a 8 , entre 28 y $35^{\circ} \mathrm{C}$, y en concentraciones de $1 \%(\mathrm{p} / \mathrm{v})$ de $\mathrm{NaCl}$. Asimismo, se hallaron rizobios con capacidad de crecimiento en condiciones que se consideran adversas y que sugieren una mayor flexibilidad fisiológica y capacidad de adaptación al ambiente. Se lograron recuperar simbiontes de $D$. virgatus tolerantes a una temperatura de $40^{\circ} \mathrm{C}$, que constituyen un material valioso como potenciales candidatos para ser considerados en programas de selección de rizobios inoculantes, ya que la termotolerancia es una característica importante para las zonas del centro y norte de nuestro país.

En relación con las características genotípicas, el análisis del germoplasma asociado a $D$. virgatus evidenció una escasa diversidad en los simbiontes locales y los aislamientos seleccionados fueron identificados como Ensifer spp sobre la base de su genotipo.

Los ensayos de inoculación de plantas que se realizaron en cámara de crecimiento de cultivo y a campo mostraron características simbióticas promisorias para la cepa termotolerante 8L12.1. La calidad de la simbiosis, que se estimó a través de la MS de las plantas, permitió seleccionar el aislamiento 8L12.1 Ensifer sp. como un candidato adecuado para una evaluación a campo en diferentes condiciones edafoclimáticas. Los resultados mostraron que cepas de rizobios nativas inoculadas en las semillas promovieron un mejor comporta- miento agronómico, y enfatizaron la importancia de la inoculación para lograr un establecimiento exitoso y crecimiento inicial adecuado de las plantas de $D$. virgatus en condiciones de campo.

\section{BIBLIOGRAFÍA}

Amarger N. 2001. Rhizobia in the field. Adv. Agron. 73: 109-168.

Bahnisch GA, Date RA, Brandon NJ, Pittaway P. 1998. Growth responses of Desmanthus virgatus to inoculation with Rhizobium strain CB3126 I. A pot trial with 8 clay soils from central and southern Queendsland. Trop. Grasslands. 32: 13-19.

Becerra Stiefel AC, Date RA, Brandon NJ. 1998. Survival of rhizobia on seed of Desmanthus virgatus stored at different temperatures. Trop. Grasslands. 32 (2): 28-33.

Beyhaut E, Tlusty B, van Berkum P, Graham PH. 2006a. Rhizobium giardinii is the microsymbiont of Illinois bundleflower (Desmanthus illinoensis (Michx.) Macmillan) in midwestern prairies. Can. J. Microbiol. 52: 903-907.

Beyhaut E, DeHaan LR, Byun JL, Sheaffer CC, Graham PH. 2006b. Response to inoculation in Illinois bundleflower. Can. J. Plant. Sci. 86: 919-926.

Brandon NJ, Date RA, Clem RL, Roberson BA, Graham TWG. 1998. Growth responses of Desmanthus virgatus to inoculation with Rhizobium strain CB3126 II. A field trial at 4 sites in south-east Queendsland. Trop. Grasslands. 32: 20-27.

Bray RH, Kurtz LT. 1945. Determination of total, organic and available forms of phosphorus in soils. Soils Sc. 59: 39-45.

Burt RF. 1993. Demanthus: A tropical and subtropical forage legume. Part. I General Review Herbage Abstracts. 63: 401-413. 
Cabrera AL. 1994. Regiones fitogeográficas argentinas. Enciclopedia Argentina de Agricultura y Jardinería. Tomo II, fascículo 1. (Ed. ACME). Bs As. 85 pp.

Calado TB, Cunha MVD, Teixeira VI, Santos MVFD, Cavalcanti HS, Lira CC. 2016. Morphology and productivity of” jureminha” genotypes (Desmanthus spp.) under different cutting intensities. Revista Caatinga, 29(3): 742-752. http://dx.doi.org/10.1590/1983-21252016v29n326rc.

Carranca C. 2013. Legumes: Properties and symbiosis. En: Camisão AH, Pedroso CC, eds. Symbiosis: Evolution, Biology and Ecological Effects. Nova Science Publishers, New York. Pp. 67-94.

Catroux G, Hartmann A, Revellin C. 2001. Trends in rhizobial inoculant production and use. Plant Soil. 230: 21-30. doi: 10.1023/A:1004777115628.

Centro Internacional de Agricultura Tropical (CIAT). 1988. Simbiosis leguminosa-rizobio. Manual de métodos de evaluación, selección y manejo agronómico. (Ed. Proy. CIAT-UNDP). Colombia. 178 pp.

Clem RL, Hall TJ. 1994. Persistence and productivity of tropical pasture legumes on three cracking clay soils (Vertisols) in north-eastern Queensland. Aust. J. Exp. Agr. 34: 161171.

Date RA. 1991. Nitrogen fixation in Desmanthus: strain specificity of Rhizobium and responses to inoculation in acidic and alkaline soil. Trop. Grasslands. 25: 47-55.

de Lajudie P, Willems A, Pot B, Dewettinck D, Maestrojuan G, Neyra M, Collins MD, Dreyfus B, Kersters K, Gillis M. 1994. Polyphasic taxonomy of rhizobia: Emendation of the genus Sinorhizobium and description of Sinorhizobium meliloti com. nov.; Sinorhizobium saheli sp. nov.; and Sinorhizobium teranga sp. nov. Int. J. Syst. Bacteriol. 44: 715-733.
Deaker R, Roughley R J, Kennedy IR. 2004. Legume seed inoculation technology-a review. Soil Biol. Biochem. 36(8): 1275-1288. doi:10.1016/j.soilbio.2004.04.009.

Di Rienzo JA, Casanoves F, Balzarini MG, González L, Tablada M, Robledo CW. 2018. InfoStat (versión 2018). [Software de cómputo] Córdoba, Argentina: Grupo InfoStat, FCA, Universidad Nacional de Córdoba. URL: http://www.infostat.com.ar

Ferreira L, Sánchez-Juanes F, García-Fraile P, Rivas R, Mateos PF, Martínez-Molina E, González-Buitrago JM, Velázquez E. 2011. MALDI-TOF mass spectrometry is a fast and reliable platform for identification and ecological studies of species from family Rhizobiaceae. PLoS One 6:e20223. doi: 10.1371/ journal.pone.0020223.

Fischbach JA, Peterson PR, Ehlke NJ, Wyse DL, Sheaffer CC. 2005. Illinois bundleflower forage potential in the upper midwestern USA: II. Forage Quality. Agron. J. 97: 895-903. doi: 10.2134/agronj2004.0139.

Fontenele ACF, Aragão WM, de Albuquerque Rangel J.H, Almeida SA. 2009. Leguminosas tropicais: Desmanthus virgatus (L.) Willd. uma forrageira promissora. R. Bras. Agrociência 15 (1-4) 121-123. doi: 10.18539/ CAST.V15I1-4.1998.

Fornasero LV, Del Papa MF, López JL, Albicoro FJ, Zabala JM, Toniutti MA, Pensiero JF, Lagares A. 2014. Phenotypic, Molecular and Symbiotic Characterization of the Rhizobial Symbionts of Desmanthus paspalaceus (Lindm.) Burkart That Grow in the Province of Santa Fe, Argentina. PLoS ONE 9(8): e104636. doi:10.1371/journal. pone. 0104636 .

Fornasero LV, Toniutti MA, Zuber NE. 2018. Caracterización fenotípica y genotípica de rizobios simbiontes de Macroptilium erythroloma recuperados de suelos de las provincias de Corrientes y Santa Fe. Ciencia del Sue- 
lo. Revista de la Asociación Argentina de la Ciencia del Suelo. 36 (2): 62-73. http://www. suelos.org.ar/publicaciones/v36n2-html/pdf/ v36n2a06.pdf

Gardiner CP. 2016. Developing and commercializing new pasture legumes for clay soils in the semi-arid rangelands of northern Australia: the new Desmanthus cultivars JCU 1-5 and the Progardes story. In: Lazier JR, Ahmad N (eds.) Tropical Forage Legumes: harnessing the potential of Desmanthus and other genera for heavy clay soils. CABI, Wallingford, UK, pp. 283-304. http://dx.doi. org/10.1079/9781780646282.0000.

Gardiner C, Kempe N, Hannah I, McDonald J. 2013. PROGARDES TM: a legume for tropical/subtropical semi-arid clay soils. Trop. Grasslands. 1: 78-80. Doi: 10.17138/ TGFT(1)78-80.

Garrity GM, Bell JA, Lilburn, T. 2005. The revised road map to the manual. In: Brenner DJ, Krieg NR, Staley JT, Garrity GM, eds. Bergey's Manual of Systematic Bacteriology, 2ndEd. Vol. 2. The Proteobacteria. Springer, New York. pp. 159-187.

Graham PH. 1992. Stress tolerance in Rhizobium and Bradyrhizobium, and nodulation under adverse soil conditions. Can. J. Microbiol. 38: 475-484.

Helrich K. 1990. Official methods of analysis of the Association of Official Analytical Chemists. (15th ed.) Association of Official Analytical Chemists Inc. Arlington, Virginia, Estados Unidos de América.

Hernández-Forte I, Nápoles-García MC, Rosales-Genqui PR, Ramírez-Pedroso JF, Ponte-Betancourt S. 2017. Tolerancia a la acidez de rizobios provenientes de nódulos de Canavalia ensiformis. Cultivos Tropicales, 38(3): 55-57.https://www.redalyc.org/articulo. oa?id=193253129007.
Hopkinson JM, English BH. 2004. Germination and hardseededness in Desmanthus. Trop. Grasslands. 38: 1-16. http://www.tropicalgrasslands.info/public/journals/4/Historic/ Tropical\%20Grasslands\%20Journal\%20archive/PDFs/Vol_38_2004/Vol_38_01_2004_ pp01_16.pdf

Howieson J, Ballard R. 2004. Optimising the legume symbiosis in stressful and competitive environments within southern Australia - some contemporary thoughts. Soil Biol. Biochem. 36: 1261-1273. doi: 10.1016/j. soilbio.2004.04.008.

Illarze Dive G. 2011. Eficiencia simbiótica entre Desmanthus spp. y rizobios nativos del Uruguay. Tesis de la Licenciatura en Ciencias Biológicas. Fac. Ciencias. Univ. República. Uruguay. 50pp.

Instituto Nacional Tecnología Agropecuaria. Estación Experimental Agropecuaria Rafaela. (INTA. EEA Rafaela). 1991. Carta de suelos de la República Argentina - Hojas, 3160 -26 y 25. Esperanza - Pilar. Santa Fe.

Jarvis BDW, van Berkum P, Chen WX, Nour SM, Fernyez MP, Cleyet-Marel JC, Gillis M. 1997. Transfer of Rhizobium loti, Rhizobuim huakuii, Rhizobium ciceri, Rhizobium mediterraneum and Rhizobium tianshanense to Mesorhizobium gen. nov. Int. J. Syst. Bacteriol. 47 (3): 895-898.

Jones RM, Clem RM. 1997. The role of genetic resources in developing improved pastures in semi-arid and subhumid northern Australia. Trop. Grasslands. 31: 315-319. http://www. tropicalgrasslands.info/public/journals/4/Historic/Tropical\%20Grasslands\%20Journal\%20 archive/PDFs/Vol_31_1997/Vol_31_04_97_ pp315_319.pdf 
Jones RM, Brandon NJ. 1998. Persistence and productivity of eight accessions of Desmanthus virgatus under a range of grazing pressures in subtropical Queensland. Trop. Grasslands 32: 145-152. http://www.tropicalgrasslands. info/public/journals/4/Historic/Tropical\%20 Grasslands\%20Journal\%20archive/PDFs/ Vol_32_1998/Vol_32_03_98_pp145_152.pdf

Jones RM, Bishop HG, Clem RL, Conway MJ, Cook BG, Moore K, Pengelly BC. 2000. Measurements of nutritive value of a range of tropical legumes and their use in legume evaluation. Trop. Grasslands. 34: 78-90. http://tropicalgrasslands.info/public/journals/4/Historic/Tropical\%20Grasslands\%20 Journal\%20archive/PDFs/Vol_34_2000/ Vol_34_02_00_pp78_90.pdf

Jordan DC. 1984. Family III. Rhizobiaceae Conn 1938. En: Krieg NR y Holt JG, eds. Bergey's Manual of Systematic Bacteriology. Williams and Wilkins, Baltimore, EE.UU. pp. 234-256.

Kuykendall LD, Young JM, Martínez-Romero E, Kerr A, Sawada H. 2005. Genus I. Rhizobium Frank 1889, 338AL. In: Brenner DJ, Krieg NR, Staley JT, Garrity GM, Eds. Bergey’s Manual of Systematic Bacteriology, 2nd Ed.,Vol. 2. The Proteobacteria. Springer, New York. pp. 325-340.

López RC, González LM, Ramírez R, Cordoví E, Gómez I, Castillo P. 2000. Aislamiento y caracterización de cepas nativas de Rhizobium aisladas de leguminosas pratenses en suelos afectados por la salinidad. Pastos y Forrajes. 23 (2): 129-134.

Luchetti AM. 2014. Las Leguminosas Mimosoideas de la provincia de Santa Fe. Natura Neotropicalis. 45 (1-2): 21-43.

Luckow M. 1993. Monograph of Desmanthus (Leguminosae-Mimosoideae). Syst. Bot. Monogr. 38: 1-166. The American Society of Plant Taxonomists.
Maier T, Klepel S, Renner U, Kostrzewa M. 2006. Fast and reliable maldi-tof ms-based microorganism identification. Nature Methods Application Notes. 3 (4): i-ii.

Martins LMV, Xavier GR, Neves MCP, Rumjanek NG. 1997. Características relativas ao crescimento em meio de cultura e a morfología de colônias de "Rizobio”. Embrapa Agrobiologia-Comunicado Técnico, 19. Centro Nac Pesquisa Agrobiología. 14 pp.

Ministerio de Agricultura y Ganadería (M.A.G.) 1982. Toma de muestras y Determinaciones Analíticas en Suelos y Aguas. Santa Fe. Ministerio de Agricultura y Ganadería. Dirección General de Extensión e Investigaciones Agropecuarias. Santa Fe. Argentina. 152 pp.

Pedraza R, Roncedo CS, Carrizo de Bellone S, Bellone CH, Pérez HE. 2001. Diferenciación de rizobios nativos que nodulan cuatro leguminosas forrajeras en Tucumán, Argentina. Cienc. Suelo. 19 (1): 79-81.

Pengelly BC, Conway MJ. 2000. Pastures on cropping soils: which tropical pasture legume to use? Trop. Grasslands. 34: 162-168.

Pengelly BC, Hall E, Auricht G, Bennell M, Cook BG. 2006. Identifying potential pasture species for grazing systems in the Mallee-Wimmera. Eds. Harsdorf, J. CSIRO Sustainable Ecosystems, Canberra. Australia. 109 pp.

Pensiero JF, Zabala JM. 2017. Recursos Fitogenéticos Forrajeros Nativos y Naturalizados para los Bajos Submeridionales: prospección y priorización de especies para planes de introducción a cultivo. FAVE Sección Ciencias Agrarias. 16 (1): 67-98.

Perticari A. 2006. Pasturas de alfalfa: importancia de una adecuada inoculación. II Congreso Nacional de Conservación y Uso de Forrajes. 
INTA Castelar. Buenos Aires, Argentina. Recuperado de: http://www.ipcva.com.ar/files/Pasturas\%20de\%20alfalfa\%20\%20importancia\%20 de\%20una\%20adecuada\%20inocul_.doc

Puente M, García J, Perticari A. 2011. Inoculación de leguminosas forrajeras con rizobios eficientes. IV Congreso de Producción y Uso de Forrajes. Buenos Aires, Argentina: Producir XXI y Estudio Ganadero Pergamino. Recuperado de http://www.produccionanimal. com.ar/produccion_y_manejo_pasturas/pasturas_cultivadas_alfalfa/120-inoculacion.pdf

Ramírez-Bahena MH, García-Fraile P, Peix A, Valverde A, Rivas R, Igual JM, Mateos PF, Martínez-Molina E, Velázquez E. 2008. Revision of the taxonomic status of the species Rhizobium leguminosarum (Frank 1879) Frank 1889AL, Rhizobium phaseoli Dangeard 1926AL and Rhizobium trifolii Dangeard 1926AL. $R$. trifolii is a later synonym of $R$. leguminosarum. Reclassification of the strain $R$. leguminosarum DSM 30132 (=NCIMB 11478) as Rhizobium pisi sp. nov. Int. J. Syst. Evol. Microbiol. 58: 2484-2490.

Sambrook J, Fritsch EF, Maniatis T. 1989. Molecular Cloning: A Laboratory Manual (Second Ed.). Cold Spring Harbor Laboratory Press. Cold Spring Harbor, N.Y.

Schluter PM, Harris SA. 2006. Analysis of multilocus fingerprinting data sets containing missing data. Mol. Ecol. Notes 6: 569-572.

Sinsuwongwat S, Nuntagij A, Shutsrirung A, Nomura M, Tajima S. 2002. Characterization of local rhizobia in Thailand and distribution of malic enzymes. Soil Sci Plant Nutr. 48: 719-727.

Soares BL, Ferreira PAA, Oliveira-Longatti SMD, Marra LM, Rufini M, Andrade MJBD, Moreira FMDS. 2014. Cowpea symbiotic efficiency, $\mathrm{pH}$ and aluminum tolerance in nitrogen-fixing bacteria. Scientia Agricola, 71(3): 171-180. doi:10.1590/S010390162014000300001.
Tamura K, Stecher G, Peterson D, Filipski A, Kumar S. 2013. MEGA6: Molecular Evolutionary Genetics Analysis version 6.0. Mol. Biol. Evol. 30(12): 2725-2729.

Teamtisong K. 2000. Some Physiological and molecular biology aspects of high effiency N2-fixation rhizobial strains in forage legumes. Tesis Master of Science in Biotechnology. Suranaree University of Technology. Thailand. 85 pp.

Toniutti MA, Fornasero LV, Albicoro FJ, Martini MC, Draghi W, Alvarez F, Lagares A, Pensiero JF, Del Papa MF. 2017. Nitrogen-fixing rhizobial strains isolated from Desmodium incanum DC in Argentina: Phylogeny, biodiversity and symbiotic ability. Syst Appl Microbiol. 40(5): 297-307. Doi: 10.1016/j. syapm.2017.04.004.

Versalovic M, Schneider FJ, De Bruijn J, Lupski J. 1994. Genomic fingerprinting of bacteria using repetitive sequence-based polymerase chain reaction. Methods Mol. Cell Biol. 5: 25-40.

Vincent JM. 1970. A Manual for the Practical Study of the Root-Nodule Bacteria. IBP Handbook No. 15, Blackwell Scientifc, Oxford. Visor GeoInta 2017. Disponible en http://visor.geointa.inta.gob.ar/

Walkey A, Black IA. 1934. An examination of the Degtjareff method for determining soil organic matter and proposed modification of the chromic acid titration method. Soil Sci. 37: 27-38. DOI: https://doi. org/10.1097/00010694-193401000-00003

Zabala MJ, Pensiero JF, Tomas P, Giavedoni JA. 2008. Morphological characterisation of populations of Desmanthus virgatus complex from Argentina. Trop. Grasslands. 42: 229-236.

Zuloaga FO, Morrone O. 1999. Catálogo de las plantas vasculares de la república Argentina. II. Dicotyledoneae. Monogr. Syst. Bot. Missouri Bot. Gard. 74:1-1269. 